\title{
ON THE LIMIT BEHAVIOR OF MULTI-AGENT SYSTEMS
}

\author{
Ionela Prodan, Sorin Olaru, Cristina Stoica \\ SUPELEC Systems Sciences (E3S) - Automatic Control Department, 3 rue Joliot Curie, Gif-sur-Yvette, 91190, France \\ \{Ionela.Prodan, Sorin.Olaru,Cristina.Stoica\}@supelec.fr \\ Silviu-Iulian Niculescu \\ Laboratory of Signals and Systems, CNRS-SUPELEC, 3 rue Joliot Curie, Gif-sur-Yvette, 91190, France \\ \{Ionela.Prodan,Silviu.Niculescu\}@lss.supelec.fr
}

Keywords: $\quad$ Linear Quadratic (LQ) Optimal Controller, Model Predictive Control (MPC), Multi-Agent Systems

\begin{abstract}
This paper addresses the optimal control of multiple (linear) agents in the presence of a set of adversary constraints which makes the convergence towards the "zero" relative position an infeasible task. By consequence, this fixed point of the relative dynamics is replaced by a set of fixed points with different basin of attraction or even by limit cycles. The present analysis is based on the existence of an optimum control law over a receding horizon with one step ahead constraint. The feasible explicit solution in terms of a piecewise affine control law is analyzed in order to characterize the limit behavior of an agent.
\end{abstract}

\section{Introduction}

Collision avoidance plays an important role in the context of managing multiple agents. In the same time it is known to be a difficult problem, since certain constraints are non-convex.

The goal of the present paper is to describe the limit behavior for an agent in the presence of adversary constraints. More precisely, the agent state trajectory has to avoid a convex region containing the origin in its strict interior. This region can represent an obstacle (static constraints) or another agent (dynamic constraints - leading in fact to a parametrization of the set of constraints with respect to the current state). There are many applications which are of particular interest to the present work, which include either static or dynamic constraints. Examples for a Mars rover which has to select feasible paths through an obstacle field are presented in (Shiller, 2000). Results in the case of robots with full dynamics and moving along a given path avoiding stationary obstacles are provided in (Bobrow et al., 1985). The behavior analysis for an agent near a restricted region is closely related to study the existence of fixed points or limit cycles (see (Poincaré and Magini, 1899)) and their stability properties.

Our interest in these phenomena is originated by the finite horizon formulation of Model Predictive
Control (MPC) technique including avoidance constraints for an agent. The first remark is that the presence of such constraints leads to the infeasibility of the control law around the origin (which is an equilibrium point for the autonomous system). This is an unusual formulation for the classical MPC design. To the best of the authors knowledge, all the studies on constrained MPC rely on the assumption that the origin is in the relative interior of the feasible region (Mayne et al., 2000), (Seron et al., 2002), (Bemporad et al., 2002) or on the frontier of the feasible region (Pannocchia et al., 2003).

The main contribution of this paper is to provide a description of the optimal solution of a constrained finite horizon optimization problem (originated by the predictive control formulation). A simple algebraic test can be formulated in order to verify the existence of a stable equilibrium point with the basin of attraction equivalent to the entire feasible region. Alternatively, similar conditions can be formulated in order to certify that the closed-loop behavior is unstable over the entire feasible region. This last result can be subsequently used for the design of unstable control laws (Chetaev, 1952) with the ultimate goal of "repelling" the trajectories from a certain sensitive region of the state space. However these considerations are out of the main topic of the present paper which concentrates on the algebraic conditions for exclud- 
ing limit cycles. The employed methods are specific for Piecewise Affine (PWA) systems analysis, with a geometric insight on the invariance properties of polytopic regions in the state space.

The rest of the paper is organized as follows. In Section 2 the constrained predictive control problem is formulated. Section 3 considers the unbounded interdicted region and analyzes the existence and uniqueness of a fixed point on the frontier of the feasible domain. Discussions based on the simulation results are presented in Section 4, while the conclusions are drawn in Section 5.

The following notations will be used throughout the paper. Denote $\mathbb{B}_{p}^{n}=\left\{x \in \mathbb{R}^{n}:\|x\|_{p} \leq 1\right\}$ as the unit ball of norm $p$, where $\|x\|_{p}$ is the $p$-norm of vector $x$. The spectrum of a matrix $M$ is the set of the eigenvalues of $M$, denoted by $\Lambda(M)=\left\{\lambda_{i}: i \in \mathbb{N}\right\}$. A point $x_{f}$ is a fixed point of a function $f$ if and only if $f\left(x_{f}\right)=x_{f}$ (i.e. a point identical to its own image). The boundary of a set $S$, denoted by $\partial S$ is the set of points which can be approached both from $S$ and from the outside of $S$.

\section{Problem Statement}

In the sequel, the principles of a receding horizon control problem are recalled. Let us model the behavior of an agent with a discrete time linear timeinvariant system:

$$
x_{k+1}=A x_{k}+B u_{k},
$$

where $x_{k} \in \mathbb{R}^{n}$ is the state of the agent, $u_{k} \in \mathbb{R}^{m}$ is the input signal and $A, B$ are matrices of appropriate dimensions. It is assumed that the pair $(A, B)$ is stabilizable. The state constraints describe a polytopic region $S$ in the state-space:

$$
S=\left\{x \in \mathbb{R}^{n}: \tilde{h}_{i}^{T} x \leq \tilde{k}_{i}, \quad i=1: n_{h}\right\},
$$

with $\left(\tilde{h}_{i}, \tilde{k}_{i}\right) \in \mathbb{R}^{n} \times \mathbb{R}$ and $n_{h}$ the number of halfspaces. This paper focuses on the case where $\tilde{k}_{i}>0$, meaning that the origin is contained in the strict interior of the polytopic region, i.e. $0 \in S$. The normalization of the right hand side of the inequalities (2) leads to

$$
S=\left\{x \in \mathbb{R}^{n}: h_{i}^{T} x \leq 1, \quad i=1: n_{h}\right\},
$$

with $h_{i}=\tilde{h}_{i} / \tilde{k}_{i} \in \mathbb{R}^{n}$. Such limitations arise both from for collision or obstacles avoidance problems. Note that the feasible region in the solutions space is a nonconvex region defined as the complement $\mathbb{R}^{n} \backslash S$. This implies at the modeling stage a compact representation of the obstacles and/or a safety region for an agent in terms of (3) ${ }^{1}$.

Let $x_{k+1 \mid k}$ denote the value of $x$ at time instant $k+1$, predicted upon the information available at time $k \in \mathbb{N}$. A finite receding horizon implementation of the optimal control law is typically based on the real-time construction of a control sequence $\mathbf{u}=\left\{u_{k \mid k}, u_{k+1 \mid k}, \cdots, u_{k+N-1 \mid k}\right\}$ that minimizes the finite horizon quadratic objective function:

$$
\begin{aligned}
& \mathbf{u}^{*}=\underset{\mathbf{u}}{\arg \min }\left(x_{k+N \mid k}^{T} P x_{k+N \mid k}+\sum_{i=1}^{N-1} x_{k+i \mid k}^{T} Q x_{k+i \mid k}+\right. \\
& \left.+\sum_{i=0}^{N-1} u_{k+i \mid k}^{T} R u_{k+i \mid k}\right) \\
& \text { subject to: }\left\{\begin{array}{l}
x_{k+i+1 \mid k}=A x_{k+i \mid k}+B u_{k+i \mid k} \\
x_{k+i \mid k} \in \mathbb{R}^{n} \backslash S, \quad i=1: N
\end{array}\right.
\end{aligned}
$$

Here $Q=Q^{T} \succeq 0, R \succ 0$ are positive definite weighting matrices, $P=P^{T} \succeq 0$ defines the terminal cost and $N$ denotes the prediction horizon.

It has to be mentioned that the solution of the unconstrained finite horizon problem is the well-known linear state-feedback control law:

$$
u_{k}=K_{L Q} x_{k}
$$

where $K_{L Q}$ is computed from the solution of the discrete algebraic Riccati equation. Making the assumption that the pair $(A, B)$ is stabilizable, the stabilizing LQ-optimal controller can be found before the resolution of the problem (4), closed-loop stability explicitly requiring that the state enters into a terminal region (containing the origin) at the end of the prediction horizon (Mayne et al., 2000). This considerations do not fit the present framework as long as the equilibrium point is not "approachable". Nevertheless, the stability analysis is an important issue and it will be one of the aims in the rest of the paper.

The objective is to find if the agent state either approaches a fixed point, or a periodic orbit (a "limit cycle"), or a finite set of fixed points. In a second stage we will be interested in the description of the basin of attraction and relate the analysis with classical stability results. For the sake of compactness, simplicity of notation and representation, an analysis of second order dynamical systems is proposed, i.e. the planar case. It turns out that this situation is simple enough to obtain useful results, and rich enough to gain some understanding about the difficulties in higher dimensions. Note that some of the 2-dimensional case arguments from planar geometry will not longer be valid

\footnotetext{
${ }^{1}$ A safety region can be associated to each agent and imposes that the inter-agent dynamics do not overlap each individual restriction. It is important to assure that a control action will not lead to a cycling behavior which implies energy consumption.
} 
in higher dimensions. Therefore, the generalization for the $n$-dimensional case is still an open issue.

\section{The unbounded restricted region}

The half-spaces defining the frontier of the feasible domain in (3) is considered. The LQ optimal control action is admitted as long as the trajectory does not transpass the constraints. Otherwise, the control action is modified such that the constraint is activated. Consequently, the explicit solution of an one-step ahead receding horizon optimization problem (i.e. $N=1$ in (4)) is analyzed, with the implications on the resulting autonomous agent dynamics. Figure 1 describes the regions obtained through partitioning with the imposed constraints, the hyperplane of the restricted region and the explicit MPC partitioning, respectively.

Consider the case of a single half-space, i.e $n_{h}=$ 1 in (3). The optimization problem to be solved is formulated as follows:

$$
\begin{aligned}
& u_{k}^{*}=\underset{u_{k}}{\arg \min }\left(x_{k+1}^{T} P x_{k+1}+u_{k}^{T} R u_{k}\right), \\
& \text { subject to: }\left\{\begin{array}{l}
x_{k+1}=A x_{k}+B u_{k} \\
h_{1}^{T} x_{k+1} \geq 1
\end{array}\right.
\end{aligned}
$$

After introducing $x_{k+1}$ in the cost function and the inequality constraint, the optimization problem (6) is reformulated as follows:

$$
\begin{gathered}
u_{k}^{*}=\underset{u_{k}}{\arg \min }\left(\frac{1}{2} u_{k}^{T}\left(R+B^{T} P B\right) u_{k}+x_{k}^{T} A^{T} P B u_{k}\right), \\
\text { subject to: }-h_{1}^{T} B u_{k} \leq-1+h_{1}^{T} A x_{k} .
\end{gathered}
$$

The solution of (7) is a continuous piecewise affine function of $x$, defined over a polyhedral partition $\left\{C_{j}\right\}_{j \in\{0,1\}}$ of $C \subseteq \mathbb{R}^{2}$ satisfying $C=\bigcup_{j \in\{0,1\}} C_{j}$ and for which $C_{0} \cap C_{1}$ have a relative empty intersection. Explicitly, the solution of (7) is defined as:

$$
u_{k}^{*}=F_{j} x_{k}+G_{j}, \quad \forall x_{k} \in C_{j} \text { and } j \in\{0,1\},
$$

where $F_{j}^{T} \in \mathbb{R}^{2}, G_{j} \in \mathbb{R}$. As illustrated in Figure 1, the polyhedral partitions $\left\{C_{j}\right\}_{j \in\{0,1\}}$ are defined as:

$$
\begin{aligned}
& C_{0}=\left\{x \in \mathbb{R}^{2}: h_{c}^{T} x \geq 1\right\}, \\
& C_{1}=\left\{x \in \mathbb{R}^{2}: h_{c}^{T} x<1\right\},
\end{aligned}
$$

where $h_{c} \in \mathbb{R}^{2}$ is given by the first-order KarushKuhn-Tucker (KKT) optimality conditions (Bempo$\operatorname{rad}$ et al., 2002):

$$
h_{c}^{T}=h_{1}^{T}(\underbrace{-B\left(R+B^{T} P B\right)^{-1} B^{T} P^{T} A}_{K_{L Q}}) .
$$

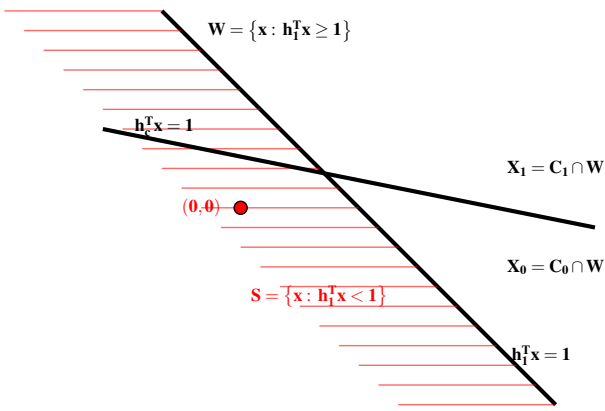

Figure 1: Exemplification of the regions determined by constraints.

Let us consider

$$
W=\left\{x \in \mathbb{R}^{2}: h_{1}^{T} x \geq 1\right\},
$$

where $0 \notin W$ (from the definition of the restricted region (3), for the case $n_{h}=1$ ). Define $X_{0}, X_{1} \subseteq \mathbb{R}^{2}$ such that, $X_{0}=C_{0} \cap W$ denotes all the state trajectories from $W$ which under the LQ dynamics (5) remain in $W$ at the next iteration and $X_{1}=C_{1} \cap W$ denotes all the state trajectories that at the next iteration transit in the restricted region. The previous definitions can be formally described as:

$$
\begin{aligned}
& X_{0}=\left\{x \in \mathbb{R}^{2}: h_{1}^{T} x \geq 1, h_{1}^{T}\left(A+B K_{L Q}\right) x \geq 1\right\}, \\
& X_{1}=\left\{x \in \mathbb{R}^{2}: h_{1}^{T} x \geq 1, h_{1}^{T}\left(A+B K_{L Q}\right) x \leq 1\right\}
\end{aligned}
$$

with $X_{0} \cup X_{1}=W$.

Remark 1 One of the optimal solutions (8) corresponds to the unconstrained optimum (5). Without any loss of generality it is assumed to be for the partition $C_{0}$ (i.e. the affine term is $G_{0}=0$ ).

Therefore the closed-loop system can be described by the following piecewise affine dynamics:

$$
x_{k+1}= \begin{cases}\left(A+B K_{L Q}\right) x_{k}, & \text { for } x_{k} \in X_{0} \\ \left(A+B F_{1}\right) x_{k}+B G_{1}, & \text { for } x_{k} \in X_{1}\end{cases}
$$

Remark 2 The existence of the constraint in the optimization problem (7) makes the origin, which represents the fixed point for the dynamics associated to the polyhedral set $X_{0}$, to reside outside the validity domain: $0 \notin X_{0}$.

Proposition 1 Consider the system (1) with the control law defined by (8). The following statements are verified:

a. All the state trajectories initiated in $x_{k} \in X_{0}$ transit in a finite time to $X_{1}$.

$b$. The dynamics associated to the polyhedral set $X_{1}$ defined by (15) will steer any point from $X_{1}$ in one step on the boundary of the feasible region, i.e. $\left\{x \in \mathbb{R}^{2}: h_{1}^{T} x=1\right\}$. 
Proof: $a$. Consider $x_{k} \in X_{0}$ such that all the future values under LQ dynamics (5) reside in $X_{0}$. As $\left(A+B K_{L Q}\right)$ is a Schur matrix it means that for any $\varepsilon \in \mathbb{R}_{+}^{*}$ there exists a $t_{\text {max }} \in \mathbb{N}$ such that $\left\|\left(A+B K_{L Q}\right)^{t_{\max }} x_{k}\right\|<\varepsilon$. But, Remark 2 states that $0 \notin X_{0}$, therefore it follows that any $x_{k}$ has to transit from $X_{0}$ in a finite time. From the definition of $X_{0}$ (13) it follows that the transit set is $X_{1}$, thus concluding the proof.

$b$. Since inside the polyhedral set $X_{0}$ (defined in (13)) the result of the optimization problem (6) corresponds to the unconstrained optimum (5) due to the fact that $h_{1}^{T} x_{k+1}>1$, it follows that the polyhedral set $X_{1}$ (14) corresponds to the constraint activation $h_{1}^{T} x_{k+1}=1$. As a consequence, for any $x_{k} \in X_{1}$ one has $x_{k+1}$ on the boundary of the feasible region, thus concluding the proof.

Remark 3 Note that a consequence of Proposition $1 . b$ is that one of the eigenvalues of the state matrix associated to $X_{1}$ is 0 since the dynamics of $X_{1}$ represent a projection on a " $n-1$ " dimensional set (i.e. on $\partial S$, with $n_{h}=1$ ).

Taking into account the above remarks, the forthcoming study is concentrated on the polyhedral set $X_{1}$. In particular, the aim is to define simple mathematical conditions for the existence and uniqueness of a stable fixed point on the frontier of the feasible domain.

Therefore, the qualitative behavior of the agent (1) is determined by the pattern of its fixed points, as well as by their stability properties. One issue of practical importance is whether the fixed point $x_{f}$ associated to the polyhedral region $X_{1}$ is stable or unstable and whether $x_{f}$ is included in $X_{1}$ or $X_{0}$.

The following theorem is stated as a main result for defining simple and sufficient algebraic conditions for the existence and uniqueness of a stable/attractive fixed point of system (15).

Theorem 1 Consider the discrete-time system (1) in closed-loop, with the optimal solution (8) of the optimization problem (6).

If there exists $V \in \mathbb{R}^{2 \times 2}$ with nonnegative elements such that

$$
\begin{gathered}
{\left[\begin{array}{r}
-h_{1}^{T} \\
h_{c}^{T}
\end{array}\right]\left(A+B F_{1}\right)=V\left[\begin{array}{r}
-h_{1}^{T} \\
h_{c}^{T}
\end{array}\right],} \\
\text { and } \\
(V-I) \mathbf{1}<-\left[\begin{array}{r}
-h_{1}^{T} \\
h_{c}^{T}
\end{array}\right] B G_{1},
\end{gathered}
$$

with $h_{c}$ defined by (11) and

$F_{1}=-G_{1} h_{1}^{T} h_{c}-\left(R+B^{T} P B\right)^{-1} B^{T} P A$,

$G_{1}=\left(R+B^{T} P B\right)^{-1} B^{T} h_{1}\left(h_{1}^{T} B\left(R+B^{T} P B\right)^{-1} B^{T} h_{1}\right)^{-1}$,

then we have the following properties with respect to $x_{f}=\left(I-\left(A+B F_{1}\right)\right)^{-1} B G_{1}$ : a. If $x_{f} \in X_{1}$, then $x_{f}$ is a unique stable fixed point with a basin of attraction $W$ defined by (12).

b. If $x_{f} \notin X_{1}$, then the closed-loop dynamics are globally unstable in $W$ with $x_{f}$ an unstable fixed point. Moreover, all the trajectories transit to infinity along the boundary of the feasible region.

Proof: The first-order Karush-Kuhn-Tucker (KKT) optimality conditions (Bemporad et al., 2002) provides the construction of $F_{1}$ and $G_{1}$. Consider the polyhedral set $X_{1}$ described as $X_{1}=C_{1} \cap W$, where the set $W$ defined in (12) is invariant with respect to the piecewise affine dynamics (15) (the set $W$ is composed by two regions $X_{0}, X_{1}$, which do not transit outside the set, see Proposition 1). Using Lemma 1 (see Appendix) we prove that $C_{1}$ defined in (10) is a positively invariant set with respect to the dynamics $x_{k+1}=\left(A+B F_{1}\right) x_{k}+B G_{1}$ from (15). Therefore, taking into account that the set $X_{1}$ is the intersection between two invariant sets, results that $X_{1}$ is also a positively invariant set.

a. Now, let us consider the case where the fixed point $x_{f}$ is contained by the invariant set $X_{1}$. Since the dynamics $x_{k+1}=\left(A+B F_{1}\right) x_{k}+B G_{1}$ associated to $X_{1}$ are affine, then the fixed point is:

- unique by the fact that over the frontier $X_{0} \cap X_{1}$ the system dynamics correspond to the closed-loop dynamics $x_{k+1}=\left(A+B K_{L Q}\right) x_{k}$ which admits a unique fixed point on the origin. But $0 \notin X_{1}$ and thus $\{1\} \notin \Lambda\left(A+B F_{1}\right)$.

- stable by the fact that the dynamics in $X_{1}$ contain an eigenvalue at the origin (see Remark 3) while the second eigenvalue is inside the unit disc as a consequence of the invariance and continuity.

The local attractivity in $X_{1}$ is completed with the attractivity in $W=X_{0} \cup X_{1}$ as a consequence of Proposition 1.

$b$. Let us consider the point $z_{k} \in \mathbb{R}^{n}$ such that

$$
z_{k} \in\left\{x \in \mathbb{R}^{2}: h_{1}^{T} x_{k}=1\right\} \cap\left\{x \in \mathbb{R}^{2}: h_{c}^{T} x_{k}=1\right\},
$$

with $h_{c}$ given by (11). In order to establish that in the case where the affine dynamics of $X_{1}$ is unstable, all the trajectories converge to infinity, it suffices to prove that all future values starting from $z_{k}$ as in (18) will transit in $X_{1}$ on the boundary of the feasible region. We prove this by contradiction.

Assume that $z_{k}$ will transit in one step in $X_{0}$ (i.e. $z_{k+1} \in X_{0}$ ). Since by its definition $z_{k} \in X_{1}$ it follows from Proposition 1.b that $z_{k+1}$ is on the boundary of the feasible region. Consequently we state the following relations:

$$
\begin{gathered}
h_{1}^{T} z_{k}=1, \\
h_{1}^{T} z_{k+1}=h_{1}^{T} A_{L Q} z_{k}=1,
\end{gathered}
$$


where $A_{L Q}=A+B K_{L Q}$ denotes the closed-loop dynamics of region $X_{0}$. Consequently, at the next iteration we have that

$$
h_{1}^{T} z_{k+2}>1 .
$$

The Cayley-Hamilton theorem states that replacing $A_{L Q} \in \mathbb{R}^{2 \times 2}$ in the characteristic polynomial yields to $A_{L Q}^{2}+c_{1} A_{L Q}+c_{2} I=0$, which is equivalent to

$$
A_{L Q}^{2}=-c_{1} A_{L Q}-c_{2} I
$$

with $c_{1}, c_{2} \in \mathbb{R}$ and

$$
c_{1}+c_{2}<1 .
$$

Introducing (22) and (23) in (21) it results that

$$
h_{1}^{T} z_{k+2}=h_{1}^{T}\left(-c_{1} A_{L Q}-c_{2} I\right) z_{k}=-\left(c_{1}+c_{2}\right) .
$$

We reach a contradiction as long as (21) is satisfied. Subsequently, we have shown by contradiction that $z_{k+1} \in X_{0}$ is false, therefore all future values starting from $z_{k}$ will transit in $X_{1}$.

Let there be $V$ and $\Lambda$ the Jordan decomposition $V \Lambda V^{-1}=A+B F_{1}$. Remark 3 states that one of the eigenvalues of the state matrix is 0 , allowing to write the dynamics associated to the set $X_{1}$ :

$$
x_{k+1}=V \Lambda V^{-1} x_{k}+B G_{1} .
$$

After elementary algebraic operations it results that

$$
\left[\begin{array}{l}
y_{1_{k+1}} \\
y_{2_{k+1}}
\end{array}\right]=\left[\begin{array}{ll}
0 & \\
& \lambda
\end{array}\right]\left[\begin{array}{l}
y_{1_{k}} \\
y_{2_{k}}
\end{array}\right]+\left[\begin{array}{l}
\delta_{1} \\
\delta_{2}
\end{array}\right]
$$

where $y_{k}=\left[\begin{array}{l}y_{1_{k}} \\ y_{2_{k}}\end{array}\right]=V^{-1} x_{k},\left[\begin{array}{l}\delta_{1} \\ \delta_{2}\end{array}\right]=V^{-1} B G_{1}$ and $\lambda$ is the nonzero eigenvalue of $\Lambda$. After $k$ iterations the following relations are obtained:

$$
\begin{aligned}
& y_{1_{k+1}}=\delta_{1} \\
& y_{2_{k+1}}=\lambda^{k} y_{2_{0}}+\sum_{i=0}^{k-1} \lambda^{i} \delta_{2}
\end{aligned}
$$

Using (27) we observe that as long as $\lambda \geq 1$ the distance between $x_{f}$ and $z_{k}$ expands at the next iteration, that is

$$
\left\|z_{k}-x_{f}||=|\lambda| \cdot\right\| z_{k+1}-x_{f} \| .
$$

In addition, if there exists $V \in \mathbb{R}^{2 \times 2}$ such that the conditions (16), (17) are satisfied, $X_{1}$ is an invariant set. Therefore, any point from $W$ has a trajectory that diverges from $x_{f}\left(x_{f} \notin X_{1}\right)$. Finally, it results that all the trajectories transit to infinity along the boundary of the feasible region.

\section{Simulation results}

Consider an agent in two spatial dimensions with the dynamics described by:

$$
A=0.9\left[\begin{array}{cc}
\cos \left(\frac{\pi}{4}\right) & -\sin \left(\frac{\pi}{4}\right) \\
\sin \left(\frac{\pi}{4}\right) & \cos \left(\frac{\pi}{4}\right)
\end{array}\right], B=\left[\begin{array}{l}
1 \\
1
\end{array}\right]
$$

where $\left[\begin{array}{ll}x & y\end{array}\right]^{T}$ and $u$ are the state and the input of the agent, respectively. The components of the state are the position coordinates of the agent. The pair $(A, B)$ is stabilizable. The tunning parameters of the optimization problem (7) are

$$
P=\left[\begin{array}{ll}
1.32 & 0.08 \\
0.08 & 2.32
\end{array}\right], R=1
$$

a. Consider the set of state constraints as defined in (3) with $h_{1}^{T}=\left[\begin{array}{ll}-0.1 & 0.01\end{array}\right]^{T}$ and $n_{h}=1$. Solving the optimization problem (7), an explicit solution defined over two polyhedral regions is obtained (Figure 2.a). The optimization problem is feasible since the agent trajectory satisfies the imposed constraints, Figure 2.b. We obtained $V=\left[\begin{array}{cc}0 & 0 \\ 0.16 & 0.8\end{array}\right]$ such that the algebraic conditions (16) and (17) defined in Theorem 1 are verified. Figure 3.a depicts the region $X_{0}$ corresponding to the unconstrained control action and the invariant set $X_{1}$ for which condition the $\left\{x \in \mathbb{R}^{n}: h_{1}^{T} x \geq 1\right\}$ is saturated. In this particular case, $X_{1}$ has a stable dynamics and contains the associated stable fixed point $x_{f}=\left[\begin{array}{ll}-5.18 & 2.96\end{array}\right]^{T}$.

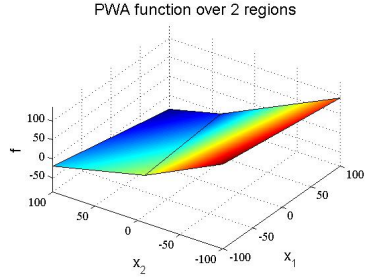

(a)

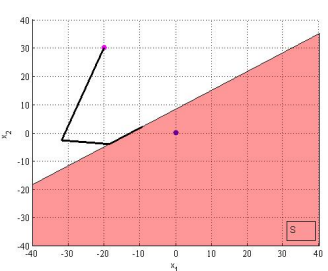

(b)
Figure 2: (a) The optimal explicit solution. (b) The evolution of the agent state trajectory which satisfies the constraints described by the half-space.

b. Consider the set of state constraints as defined in (3) with $h_{1}^{T}=\left[\begin{array}{ll}0.31 & 0.42\end{array}\right]^{T}$ and $n_{h}=1$. We obtained $V=\left[\begin{array}{cc}0 & 0 \\ 0.16 & 3.01\end{array}\right]$ such that the algebraic conditions (16) and (17) defined in Theorem 1 are verified. Figure $3 . b$ illustrates the region $X_{0}$ corresponding to the unconstrained control action and the region $X_{1}$ corresponding to the saturation of $\left\{x \in \mathbb{R}^{n}: h_{1}^{T} x \geq 1\right\}$. In this case, $X_{1}$ has an unstable dynamics and the associated unstable fixed point $x_{f}=\left[\begin{array}{ll}14.05 & -8.03\end{array}\right]^{T}$ is in $X_{0}$. The conditions of Theorem 1.b are fulfilled, therefore all the points transit to infinity along the boundary of the feasible region, Figure 3.b.

For any hyperplane tangent to the circle (Figure 3) there exists $V \in \mathbb{R}^{2 \times 2}$ with nonnegative elements such 
that (16) and (17) defined in Theorem 1 are verified.

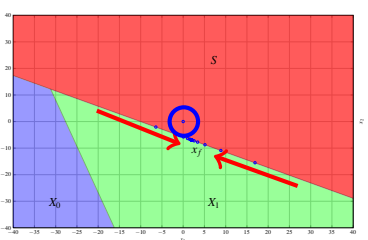

(a)

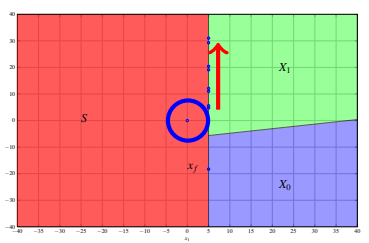

(b)
Figure 3: (a) Exemplification for the existence of a unique stable fixed point on the boundary of the feasible region. (b) Exemplification for the case where all the points transit to infinity.

\section{Conclusions}

The paper presents and develops a set of conditions for the optimality of a dynamic motion planning of an agent in the presence of a set of adversary constraints. This type of constraints are unusual because they make the agent trajectory converging to origin to be infeasible. Since the origin is normally a stable point, these conditions may generate a limit cycle. Consequently, simple algebraic conditions for the existence and uniqueness of a stable fixed point on the boundary of the feasible region represent the main result of this paper.

\section{REFERENCES}

Bemporad, A., Morari, M., Dua, V., and Pistikopoulos, E. (2002). The explicit linear quadratic regulator for constrained systems. Automatica, 38(1):3-20.

Bitsoris, G. (1988). On the positive invariance of polyhedral sets for discrete-time systems. Systems \& Control Letters, 11(3):243-248.

Bobrow, J., Dubowsky, S., and Gibson, J. (1985). Time-optimal control of robotic manipulators along specified paths. The International Journal of Robotics Research, 4(3):3.

Chetaev, N. (1952). On the instability of equilibrium in some cases where the force function is not maximum. Prikl. Mat. Mekh, 16(1).

Mayne, D., Rawlings, J., Rao, C., and Scokaert, P. O. (2000). Constrained model predictive control: Stability and optimality. Automatica, 36:789814.

Pannocchia, G., Wright, S., and Rawlings, J. (2003). Existence and computation of infinite horizon model predictive control with active steady-state input constraints. IEEE Transactions on Automatic Control, , 48(6):1002-1006.

Poincaré, H. and Magini, R. (1899). Les méthodes nouvelles de la mécanique céleste. Il Nuovo $\mathrm{Ci}$ mento (1895-1900), 10(1):128-130.

Seron, M., De Dona, J., and Goodwin, G. (2002). Global analytical model predictive control with input constraints. In Proceedings of the 39th IEEE Conference on Decision and Control, volume 1, pages 154-159, Sydney, Australia.

Shiller, Z. (2000). Obstacle traversal for space exploration. In Robotics and Automation, 2000. Proceedings. ICRA'O0. IEEE International Conference on, volume 2, pages 989-994.

\section{Acknowledgements}

The authors would like to thank anonymous reviewers for their useful comments and remarks. The research of Ionela Prodan is financially supported by the EADS Corporate Foundation (091-AO09-1006).

\section{APPENDIX-Set Invariance}

We use as an instrumental result the following lemma, which is an adaptation for affine systems of the Proposition 2 from (Bitsoris, 1988).

\section{Lemma 1 Consider the polyhedral set}

$$
Z(H, K)=\left\{x \in \mathbb{R}^{n}: H x \leq K\right\},
$$

with $(H, K) \in \mathbb{R}^{r \times n} \times \mathbb{R}^{r}$. If there exists $V \in \mathbb{R}^{r \times r}$ with nonnegative elements such that

$$
\begin{gathered}
H \Phi=V H \quad \text { and } \\
(V-I) K+H \Gamma \quad \leq 0,
\end{gathered}
$$

then $Z(H, K)$ is a positively invariant set with respect to the affine dynamics $x_{k+1}=\Phi x_{k}+\Gamma$.

Proof: Suppose $x_{k} \in Z$. We want to prove that $x_{k+1} \in Z$. By explicitly replacing

$$
H x_{k+1}=H\left(\Phi x_{k}+\Gamma\right) \stackrel{(31)}{=} V H x_{k}+H \Gamma,
$$

and taking into account the hypothesis that $H x_{k} \leq K$ and $V$ has nonnegative elements it follows that

$$
H x_{k+1} \leq V K+H \Gamma .
$$

with $V K+H \Gamma=(V-I) K+H \Gamma+K \leq K$ which can then be verified by (32). 